\title{
L'énonciation conjointe : trace et ressource de la construction collaborative du discours
}

\author{
André, Virginie \\ ATILF \\ Université de Lorraine \& CNRS \\ Virginie.Andre@univ-lorraine.fr
}

\section{Introduction}

Les interactions verbales ne se déroulent pas de façon chaotique (Mondada, 1999a). Les participants s'ajustent en permanence, en fonction de leurs interlocuteurs, de leurs productions langagières notamment, ainsi qu'en fonction des nombreux autres éléments de la situation de communication (Hymes, 1972). Ces ajustements sont donc à la fois situationnels et communicationnels (Charaudeau, 1995). En d'autres termes, lorsque les locuteurs s'engagent dans une interaction verbale, ils produisent des formes linguistiques et interactionnelles adéquates (ajustements communicationnels), en fonction d'une certaine connaissance de la situation à laquelle ils prennent part (ajustements situationnels). Ces ajustements permanents participent à la construction collaborative du discours.

Parmi les phénomènes langagiers participant à ou illustrant la construction collaborative du discours, nous analyserons ici ce que nous appelons les énonciations conjointes. Dans un premier temps, nous présenterons le cadre dans lequel s'inscrit cette étude afin de préciser notre approche sociolinguistique des interactions verbales. Dans un deuxième temps, nous préciserons notre définition de l'énonciation conjointe. Nous présenterons ensuite le corpus de travail utilisé pour cette étude ainsi que la façon dont les données ont été repérées avant d'être analysées. Enfin, dans la quatrième partie de cet article, nous analyserons des exemples d'énonciation conjointe apparaissant à la fois comme des traces et des ressources de la construction collaborative du discours.

\section{Sociolinguistique des interactions verbales}

Notre analyse de la construction collaborative du discours, et plus précisément dans le cadre de cette étude des énonciations conjointes, nous amène à faire appel à une approche sociolinguistique des interactions verbales. Cette approche sociolinguistique appréhende le discours, à la fois comme construit de manière séquentielle, c'est-à-dire construit tour par tour par les interactants et comme influencé par de nombreux éléments extralinguistiques, préexistants à l'interaction et apparaissants lors de l'interaction. En d'autres termes, nous proposons une approche qui associe plusieurs outils d'analyse, notamment l'analyse conversationnelle, l'analyse de discours et la sociolinguistique, afin de saisir toute la complexité des interactions verbales et de la construction du discours. Notre démarche s'apparente à celle de KerbratOrecchioni $(2005: 21)$ qui propose une « approche éclectique ${ }^{1} »$ :

Il me semble plus intéressant et rentable de concilier ce qui est conciliable, et de voir le parti que l'on peut tirer du croisement de propositions venant de paradigmes différents. La description est alors moins « pure » - si tant est qu'une approche puisse jamais être chimiquement pure : aucun modèle n'est constitué exclusivement de notions « endogènes », car les concepts essaiment, émigrent, traversent les frontières des écoles et même des 
disciplines, comme on l'a vu pour le concept d'interaction ; mais elle est plus riche, et même parfois plus « juste » car le métissage théorique n'est pas seulement un luxe, c'est dans certains cas une nécessité.

Nous empruntons aux approches interactionnelles, conversationnelles et discursives, les outils nécessaires pour analyser l'apparition de certains phénomènes langagiers et l'analyse sociolinguistique nous invite à repérer les facteurs extralinguistiques pertinents intervenant dans la construction du discours. Ces facteurs extralinguistiques sont notamment : l'identité des locuteurs, leurs rôles conversationnels, leur statut, leurs connaissances paratgées; les objectifs de l'interaction; son histoire conversationnelle; son épaisseur historique (implications sociales, individuelles, économiques, cuturelles ou encore politique).

Notre approche est nécessairement pluridisciplinaire et multidimensionnelle dans la mesure où elle tente de prendre en compte, dans l'analyse des productions langagières des locuteurs, l'ensemble des éléments exerçant une influence sur ces productions et sur le déroulement des interactions. Nous pouvons illustrer ces multiples influences par le schéma suivant :

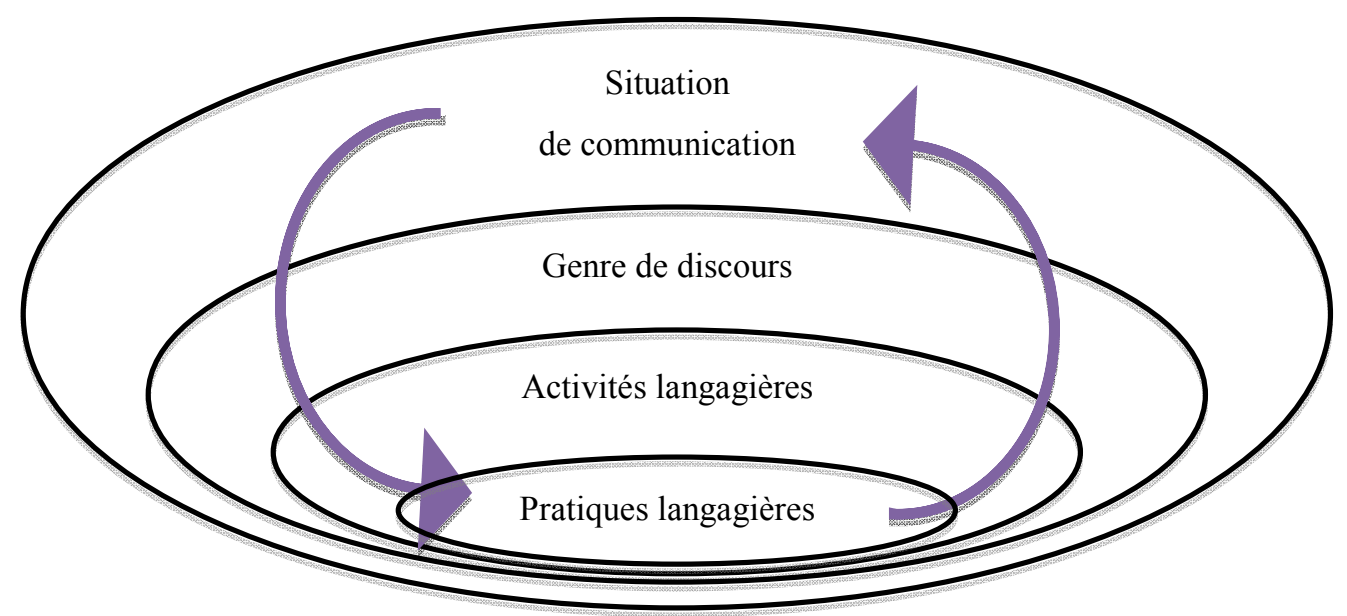

Schéma 1: Intrications et interinfluences entre pratiques langagières, activités langagières, genre de discours et situation de communication

Ce schéma tente de montrer que les pratiques langagières, c'est-à-dire les productions linguistiques et interactionnelles des locuteurs, sont actualisées afin d'accomplir des activités langagières prenant place dans un genre de discours particulier (conversation, réunion de travail, conférence, débat, etc.) et dans une situation de communication toujours particulière. Ces quatre éléments encerclés dans le schéma sont tous intriqués les uns avec les autres, chacun conditionnant l'actualisation ou la réalisation de l'autre. Les flèches de ce schéma montrent que les influences sont réciproques. Le premier mouvement de ces influences (représenté par la flèche descendante) part de la situation pour aller vers les pratiques. Il illustre l'ensemble des influences des éléments extralinguistiques sur les autres éléments : le genre de discours, les activités langagières et les pratiques langagières. Ce mouvement traverse chacun des éléments du schéma pour montrer que chacun exerce une influence sur le suivant. Le second mouvement (représenté par la flèche ascendante), montre que les influences que nous venons d'évoquer sont 
réciproques, c'est-à-dire que les pratiques langagières influencent les activités langagières (leur réalisation et leur réussite, par exemple) et que ces deux éléments redéfinissent le genre de discours (son script, par exemple) ainsi que la situation de communication de départ (la relation entre les interactants, leurs rôles, leurs statuts, les objectifs de l'échange, les thèmes abordés, par exemple).

Notre approche sociolinguistique des interactions verbales appréhende le discours comme profondément ancré dans le contexte social dans lequel il est produit et comme largement influencé par une multitude d'éléments rendant son analyse complexe. Nous pouvons ajouter également que les locuteurs sont sans cesse amenés à s'ajuster, comme nous l'avons précisé en introduction. Cela les amène à négocier la structure locale et le déroulement de l'interaction (Sacks et al., 1974 ; Kerbrat-Orecchioni, 2005) pour atteindre les objectifs visés par et au cours des échanges. Le discours est donc produit de façon collaborative entre les locuteurs. Nous combinons ici une analyse de type conversationnel, examinant le déroulement des interactions au fu et à mesure de la construction du discours et une analyse sociolinguistique prenant en compte le contexte dans lequel se déroulent les interactions.

Au cours de cette construction collaborative, les locuteurs s'ajustent à la fois en fonction des productions de leurs interlocuteurs et du contexte situationnel mais ils peuvent également imaginer, faire des hypothèses, prévoir ou anticiper le déroulement des interactions verbales. Les locuteurs peuvent participer conjointement à la construction du discours, en fonction de leur propre interprétation du déroulement des interactions. Cette étude s'intéressera à la mise en œuvre de ces ajustements ainsi qu'à leurs implications dans la construction collaborative du discours en analysant l'énonciation conjointe, une forme de participation aux interactions verbales.

\section{L'énonciation conjointe : un phénomène collaboratif}

Nous considérons l'énonciation conjointe comme un phénomène collaboratif dans la mesure où elle est constituée de l'enchainement de deux tours de parole, le second (la complétion) étant produit dans la continuité du premier et complète celui-ci. Nous pouvons schématiser ce phénomène de la façon suivante :

\section{Enonciation conjointe $=$}

$\diamond$ Premier locuteur : tour de parole 1

$\diamond$ Deuxième locuteur : tour de parole 2 / complétion

Nous pouvons examiner un exemple de cette construction actualisé dans notre corpus (nous le décrirons dans le point 3).

\section{Exemple 1 (À propos d'une note ministérielle)}

L1 donc tout le monde sera sur le même

L2 pied d'égalité 
Dans cet exemple, le deuxième locuteur intervient en complétant l'énoncé du premier, la complétion s'inscrit dans la construction syntaxique du premier tour de parole. Cette complétion semble donc appropriée à la fois syntaxiquement et discursivement, c'est-à-dire qu'elle respecte le cadre syntaxique ainsi que la cohérence thématique proposés par le premier locuteur. L'activité langagière (ici, informer) est ici réalisée de manière collaborative avec la contribution de deux locuteurs différents et avec un phénomène linguistique et interactionnel particulier : l'énonciation conjointe.

Dans sa description des échanges langagiers prenant la forme de paires adjacentes, Sacks (1992) définit déjà la deuxième partie d'une paire «initiation-réaction » comme une complétion possible d'un tour de parole. Sacks et al. (1974 : 702) précisent également que certaines réactions anticipent la fin du premier tour : « there are various unit-types with which a speaker may set out to construct a turn. (...) Instances of the unit-types so usable allow a projection of the unit-type under way, and what, roughly, it will take for an instance of that unit-type to be completed ». Le locuteur en cours peut voir son tour complété par l'intervention d'un autre locuteur. Ce dernier peut saisir une opportunité et produire une complétion avant la fin d'une unité de construction de tour. Sacks présente ce type de construction, qu'il appelle " collaborative sentence », comme un élément clé de la conversation.

A la suite des travaux de Sacks repris notamment par Lerner (1991), Jeanneret (1999) analyse la «coénonciation » entre les locuteurs. Elle inscrit sa réflexion, distinctement, dans deux approches : discursive et conversationnelle. Dans le cadre de la première, elle identifie ce phénomène comme une intervention dialogale, c'est-à-dire un composant monologique dans lequel interviennent deux locuteurs. Dans le cadre de l'analyse conversationnelle, Jeanneret décrit la coénonciation comme une coconstruction de tour plutôt que comme une succession de deux tours. Dans le cadre de notre étude, nous analysons l'énonciation conjointe comme la succession de deux tours de parole visant à accomplir la même activité langagière et marquant la collaboration entre les locuteurs. Nous verrons que ce phénomène est influencé par certains éléments de la situation et présente des effets pragmatiques induits par cette construction collaborative.

Jeanneret (1999) explique également que le terme coconstruction est mal choisi car il existe déjà, notamment chez Culioli (1990-1999), pour faire référence aux multiples ajustements que nous avons déjà mentionnés. En ce qui nous concerne, pour faire référence à ce processus dynamique inhérent à tout discours, nous utilisons le terme plus général de «construction collaborative ». De plus, dans une approche syntaxique, pour Jeanneret, le terme de coénonciation «a un sens plus formel » $(1995: 389)$ dans la mesure où elle rend compte d'un enchaînement syntaxique et pas seulement d'une collaboration langagière. Notre analyse montera que lorsqu'il y a enchaînement ou continuité syntaxique nous sommes en présence d'un type particulier d'énonciation conjointe mais que la continuité syntaxique n'est pas une condition à l'apparition de ce phénomène collaboratif.

Dans le cadre de sa "grammaire-pour-l'interaction ", Mondada (1999a) s'est penchée sur ces phénomènes «d'achèvement collaboratif» au sein de discours oraux interactifs. Elle analyse ces phénomènes «d'élaboration collective » comme « un terrain qui favorise la formulation d'articulations entre organisation de l'interaction, organisation grammaticale et organisation sociale » (Ibid.: 31). Lors d'une étude sur l'organisation séquentielle et interactionnelle de messages asynchrones sur Internet, comme les courriels ou les messages échangés dans des forums de discussions, Mondada fait également référence à des « enchaînements collaboratifs ", des « continuations » et des «expansions » (1999b : 25) d'une unité syntaxique. Blanche-Benveniste (2000), dans le cadre de ce qu'elle appelle les « textes à plusieurs voix ", explique également que la complétion peut être réalisée dans le prolongement syntaxique d'un énoncé tout en étant rejetée en raison de son contenu sémantique. La collaboration discursive ne marque donc pas nécessairement une convergence d'opinion entre les interactants.

Les multiples réalisations et finalités attachées à ce type de phénomène ont donné naissance à une grande variation terminologique dès les années 1970 au sein de l'analyse conversationnelle. En 1973, Jefferson évoque la notion de "collaborative sentence », déjà présente dans les années 1960 dans les cours de H. Sacks (1992) qui utilise également l'expression « collaboratively built sentence ». Cette notion sera reprise en 1999 par Jeanneret et traduite par «phrase en collaboration ». Nous rencontrons également 
d'autres appellations : « complétion du tour de parole» (André-Larouchebouvy, 1984), « locuteur collectif » (Loufrany, 1981, 1985), «duo conversationnel » ou « coopération conversationnelle » (Gumperz, 1989 ; s'inspirant des travaux de Falk en 1980), " single syntactic unit jointly produced » (Lerner, 1991), "joint construction of utterances » ou « construction conjointe d'énoncés » (Coates, 1994), "élaboration syntaxique à deux voix » (Maury-Rouan, 1998) et «formulation conjointe » (Jeanneret, 2001). Au-delà de ce foisonnement terminologique, des phénomènes différents sont souvent décrits allant de la construction syntaxique à deux locuteurs à la simple co-construction discursive.

Ce phénomène interactionnel collaboratif apparaît quel que soit le genre de discours et met en lumière la coordination des actions discursives des locuteurs. Les complétions reflètent une collaboration linguistique et praxéologique, les discours étant le fruit d'une activité conjointe (Clark, 1996). Nous nous intéressons à ces types de construction et d'enchaînement du discours dans la mesure où ils mettent également en évidence l'influence de certains éléments extralinguistiques, tels que les objectifs de la situation de communication, mais également les relations entretenues par les participants ou encore leur identité et leur rôle au sein de l'échange. Réflexivement, les énonciations conjointes, par les valeurs pragmatiques qu'elles véhiculent, participent à la création de nouveaux éléments situationnels, tels que des alliances ou des coalitions entre participants.

\section{Corpus de travail et méthodologie d'analyse des données}

Le corpus de travail utilisé dans le cadre de cette étude est composé de deux corpus que nous souhaitons distinguer en fonction de notre implication dans le recueil des données.

- Le premier corpus intitulé « Réunions » est un corpus que nous avons entièrement constitué. Il est composé de 40 heures d'enregistrement et de 330000 mots transcrits. La constitution de ce corpus a été accompagnée d'une longue enquête de terrain et de nombreux allers-retours auprès de nos informateurs qui ont permis de saisir les interactions verbales et les influences de la situation de communication. Une analyse sociolinguistique de type ethnographique a été réalisée afin de rendre intelligible l'ensemble des échanges langagiers entre les participants.

- Le second corpus est celui du projet TCOF (Traitement de Corpus Oraux en Français), disponible librement sur http://www.cnrtl.fr/corpus/tcof/ (André, Canut 2011). Nous avons utilisé les interactions entre adultes de ce corpus qui compte également de nombreuses interactions entre adultes et enfants. Le (sous)corpus utilisé pour cette étude compte également 40 heures d'enregistrements et 400000 mots transcrits (alignés texte-son avec le logiciel Transcriber $^{2}$ ). Ce corpus est composé essentiellement de données recueillies par des chercheurs et des étudiants de l'Université de Lorraine. Il est constitué de différents genres de discours (conversations, réunions de travail, entretiens, consultation médicales, conseils municipaux, réunions pédagogiques, etc.).

Nous souhaitons distinguer ces deux corpus dans la mesure où, notre implication dans la constitution et le traitement des données étant différente, notre processus d'interprétation et d'analyse des données sera nécessairement différent. En effet, nous connaissons parfaitement le corpus «Réunions », ses locuteurs, leurs relations, la finalité des réunions enregistrées, les thèmes abordés, les chantiers évoqués, etc. Ce corpus a été constitué dans l'objectif d'analyser la collaboration discursive entre les locuteurs, plus particulièrement au sein d'un collectif de travail. Notre approche sociolinguistique des interactions verbales nous a conduit naturellement à adopter une linguistique de terrain avec un investissement important notamment ici dans un environnement professionnel que nous ne connaissions pas. Boutet (1998 : 248) précise à ce sujet que « l'introduction des discours au travail comme objet d'étude des linguistes conduit (...) à une réflexion méthodologique sur le statut des matériaux à analyser, sur les méthodes d'enquête, sur la place du chercheur ». A la manière des ethnologues, nous avons enquêté, 
observé, recueilli, interrogé pour obtenir les informations nécessaires à la compréhension des discours émis lors des réunions de travail que nous avons enregistrées. Nous avons ainsi plus facilement accès à l'intelligibilité des interactions et à l'influence des éléments de la situation de communication. Nous pouvons notamment saisir les phénomènes collaboratifs actualisés pendant les échanges.

Le corpus TCOF rassemble des enregistrements et des transcriptions élaborés à la fois par les chercheurs de ce projet, dont nous faisons partie, et par les étudiants formés à la constitution de corpus. Ces données sont diffusées librement et gratuitement et sont traitées dans l'objectif d'accueillir le plus grand nombre possible d'analyses (par exemple, les membres du projet ont choisi une transcription orthographique, respectant l'orthographe standard, et une transcription assez «nue» pour être la plus lisible possible). Néanmoins, pour que le corpus TCOF puisse être utilisé dans le cadre d'une approche sociolinguistique des interactions verbales, chaque donnée primaire (enregistrement) est accompagnée d'un grand nombre de métadonnées décrivant la situation de communication. Une fiche descriptive de chaque enregistrement est remplie minutieusement et nous renseigne notamment sur le lieu et le moment de l'interaction, sur sa finalité, sur les locuteurs (leur identité personnelle, leurs relations avec les autres locuteurs, leur âge, leur profession, leur niveau d'études, leur lieu d'habitation, leur lieu de naissance, etc.). Chaque enregistrement est également accompagné d'un résumé de la situation de communication et peut être accompagné de toutes les notes que la personne qui recueille les données estime être utiles aux futur(e)s analystes de ses données. Ainsi, même si nous n'avons pas constitué entièrement ce corpus, nous avons des éléments pour comprendre les interactions et saisir les influences de la situation de communication.

Les précisions que nous apportons sur le corpus de travail nous paraissent importantes dans la mesure où le partage et la mutualisation des données sont aujourd'hui d'actualité. Nous pensons notamment au projet ORFEO (Outils et Ressources sur le Français Ecrit et Oral), soutenu par l'ANR (Agence Nationale de la Recherche) et coordonné par Jeanne-Marie Debaisieux (Lattice \& Université Sorbonne Nouvelle Paris 3), qui vise, parmi d'autres objectifs, la mutualisation des données recueillies et traitées par différents laboratoires et par différents chercheurs. Le partage des données permet de faire avancer les recherches sur le fonctionnement de la langue mais il peut également s'avérer infructueux si les données sont difficilement partageables en raison de leur intrication avec la situation dans laquelle elles ont été recueillies. Nous pouvons mentionner une exclamation prononcée par une collègue lors d'un travail collaboratif: «Ton corpus j'y comprends rien!», à propos justement de notre corpus «Réunions » (André 2011). Ainsi, notre approche sociolinguistique de la langue nous amène à mettre en place une méthodologie de travail particulière, permettant de prendre en compte l'ancrage du discours dans la situation dans laquelle il est construit. L'analyse du corpus TCOF dans le cadre de cette étude se base donc sur les éléments intelligibles des interactions, certains phénomènes interactionnels peuvent ne pas être analysés si les éléments situationnels nécessaires à l'analyse sont manquants ou partiels.

Notre méthodologie d'analyse s'est déroulée en trois parties indissociables et sans cesse entremêlées. La phase de repérage des énonciations conjointes au sein de notre corpus de travail a été associée à une analyse pragmatique et socio-interactionnelle des échanges ainsi qu'à une analyse formelle. Le repérage des énonciations conjointes est nécessairement un repérage « manuel » dans le corpus, l'automatisation de la recherche de ce type de phénomène n'est pas encore d'actualité. L'analyse est nécessairement qualitative. Elle mêle l'analyse des formes, de la gestion de l'interaction et des éléments extralinguistiques. Nous nous sommes notamment intéressée aux conditions d'apparition des énonciations conjointes et à leurs effets dans le discours.

\section{Analyse des énonciations conjointes}

L'analyse de notre corpus nous a permis d'identifier plusieurs types d'énonciations conjointes. Nous avons pu établir différentes catégorisations en fonction des formes linguistiques et interactionnelles impliquées dans la construction conjointe de l'énonciation et en fonction des objectifs visés par ce 
phénomène collaboratif (André, 2006). Nous ne pouvons pas présenter ici tous les types d'énonciations conjointes que nous avons identifiés. Nous avons choisi d'examiner, dans cet article, les énonciations conjointes :

- $\quad$ qui mettent au jour le processus de construction collaborative du discours, c'est-à-dire qui comportent des traces linguistiques et interactionnelles de cette construction collaborative ou encore qui montrent la façon dont le discours est co-produit au fur et à mesure de l'interaction ;

- qui apparaissent également comme des ressources utilisées, volontairement ou non, par les interactants pour construire collaborativement leur discours et pour donner à ce dernier des effets particuliers. Ces ressources utilisées et ces effets induits sont analysés à la lumière des éléments situationnels qui influencent les interactions, tels que l'identité des locuteurs et les objectifs ou les enjeux de la communication.

Pour illustrer ce phénomène collaboratif nous avons également choisi de concentrer notre analyse sur deux types d'énonciations conjointes :

- celles dont l'apparition de la complétion est favorisée par des indices dans le premier tour de parole ;

- celles dont l'apparition de la complétion est entièrement dépendante du second locuteur.

Notre approche sociolinguistique des interactions verbales nous permet d'identifier les facteurs extralinguistiques entrant en jeu dans la construction collaborative du discours. Nous verrons que l'apparition des énonciations conjointes peut être conditionnée par ces facteurs qu'ils soient individuels, collectifs, sociaux ou plus largement contextuels. Ces mêmes facteurs permettent également aux participants d'orienter leur interprétation du discours en train de se produire. Par exemple, au sein d'une réunion de travail, le locuteur qui complète peut être légitime pour réaliser cette action collaborative grâce à son statut au sein de l'organisation et réciproquement, l'action collaborative est accueillie et interprétée par les auditeurs en fonction de ce statut.

\subsection{Des indices linguistiques et interactionnels qui favorisent l'énonciation conjointe}

Comme nous l'avons expliqué précédemment, le premier tour de parole peut comporter des éléments linguistiques et interactionnels que le deuxième locuteur peut interpréter comme un moment opportun de prise de tour de parole. Ces indices vont favoriser l'apparition de la complétion qui peut alors être interprétée comme une aide à la construction du discours. C'est le cas dans l'exemple 2, extrait d'une conversation sur l'enfance de L2 en Algérie.

\section{Exemple 2}

L2 elle elle est née à Mostaganem < tu vois bien

L3 moi je suis née $>$

L2 la différence il y a il y a deux cents kilomètres de::

L1 entre les deux

L2 entre les deux

Dans cet exemple, L2 produit un allongement ${ }^{3}$ (« de:: ») qui semble être interprété comme une hésitation de la part de L1. Ce dernier apporte une complétion qui vient préciser les paroles de L2. L'hésitation semble donc ouvrir la possibilité pour un locuteur de compléter les propos de son interlocuteur et de contribuer à la construction du discours. La complétion est ratifiée par L2 qui la reprend. Nous rencontrons ce même procédé dans l'exemple suivant, extrait d'une réunion de travail. 


\section{Exemple 3 (A propos d'un arrêt ministériel)}

L1 (...) bon alors pour faire f- plaisir à *P2* et sur un sujet que j'avais un petit peu balayé la dernière fois hein sur sa note sur les dérogations euh aux::

L2 aux garanties minimales

L1 aux garanties minimales donc euh est paru donc le 8 janvier

Le locuteur L1 semble chercher ses mots, nous pouvons le constater d'une part par l'hésitation « euh » et par l'allongement de «aux». Ces hésitations semblent légitimer l'intervention de L2 qui complète les propos de L1. Dans cet exemple, nous pouvons relever que la complétion est réalisée par un locuteur particulier (L2 qui est aussi P2), très impliqué dans l'application de l'arrêté ministériel et qui a réalisé une note de synthèse relative à cet arrêté. La collaboration entre les locuteurs est liée à l'identité de chacun, à leur rôle au sein de l'entreprise et à leur collaboration de travail. Ce contexte permet à L2 d'anticiper la fin de l'unité constructionnelle de tour de L1. Ce dernier ratifie cette complétion par une reprise de cette dernière et poursuit son discours. L2 apporte une aide à la construction du discours de son interlocuteur.

La construction collaborative peut également se faire avec trois locuteurs différents comme dans l'exemple suivant extrait d'une conversation en famille sur le théâtre.

\section{Exemple 4}

L2 non mais euh $>$ dans la classe il y en a déjà euh trois qui v- qui v-

L1 qui veulent $<$ être veulent

L3 descendre à Paris >

L2 oui qui pense qu'à ça quoi enfin quand tu vois Charlotte elle son rêve c'est de devenir comédienne

Si nous disposons cet extrait en grille (Blanche-Benveniste, Jeanjean, 1987), nous pouvons saisir le mode de production du discours :

L2 dans la classe il y en a déjà euh trois

L1

L3

$$
\begin{array}{ll}
\text { qui v- } & \\
\text { qui v- } & \\
\text { qui } & \text { veulent }<\text { être } \\
\text { veulent }
\end{array}
$$

descendre à Paris >

La disposition en grille nous montre la façon dont le discours a été construit collaborativement par les trois locuteurs, grâce à des complétions successives. L2 a produit deux indices d'hésitation ou de recherche de termes avec « euh » puis avec le piétinement sur l'axe paradigmatique avec la répétition de « qui v- ». L1 vient en aide à L2 en apportant une complétion « qui veulent être veulent ». Cette dernière comporte également un indice d'hésitation avec la reprise de «veulent». A son tour, L3 apporte une complétion « descendre à Paris » produite à la suite du premier verbe du tour précédent (simultanément à «être veulent», le chevauchement de paroles étant marqué par les chevrons dans la transcription). Si nous nous concentrons sur les dernières lignes de la disposition en grille, nous pouvons constater l'élaboration collaborative de l'énoncé «dans la classe il y en a déjà euh trois qui veulent descendre à Paris ». 
Nous constatons d'autres traces de la construction collaborative du discours dans l'exemple suivant, extrait d'une réunion de travail.

Exemple 5 (Les locuteurs évoquent le nombre de tonnes de graviers nécessaire pour la construction d'une route du département.)

L6 ben il nous manquera les 15000 mètres cubes

L2 d' accord

L6 que l'on a défini la semaine dernière sauf que au lieu d'avoir nos 100000 tonnes de 020 concassés on en aura un petit peu moins de la moitié mais on a un excédent de 020 criblés

L2 hum hum d'accord oui parce que en 020 criblés on avait dit /

L6 150000 tonnes $\backslash$

L2 oui $\backslash$ monsieur *P4* avait dit 150000 tonnes en le traitant un peu à la chaux

L2 offre la possibilité, avec l'intonation montante avec laquelle est produit le segment « on avait dit », à un locuteur particulier (L6), désigné par le regard et un geste de la tête, de compléter son tour. Le locuteur désigné répond favorablement à cette sollicitation en complétant le tour avec l'information attendue («150000 tonnes »). L6 est sollicité par son interloctueur dans la mesure où il a une position d'expert concernant le thème abordé lors de cet extrait, c'est lui qui coordonne les travaux du chantier de l'entreprise qu'il représente. L'énonciation conjointe est réalisée par deux locuteurs détenant les informations à communiquer. Nous pouvons constater ici que les connaissances sont socialement et inégalement distribuées (Hutchins, 1995 ; Lonchamp, 2003), entre les participants à la réunion, mais partagées lors des interactions. Cette répartition inégale des connaissances entre les locuteurs explique certains phénomènes de construction collaborative du discours, notamment au sein de collectifs de travail. Un premier locuteur commence son tour de parole en fournissant les informations dont il dispose puis un second locuteur intervient pour compléter ces informations ou pour en apporter des nouvelles. Ce processus peut, comme nous l'avons constaté dans cet exemple 5, donner lieu à une énonciation conjointe.

Nous pouvons examiner un dernier exemple dans lequel la complétion semble être légitimée par une hésitation dans le premier tour de parole.

Exemple 6 (L1, directeur de l'entreprise, et L2, directeur d'un service, évoquent les futurs travaux à réaliser et le budget attribué à ces travaux.)

L1 oui non mais > toi toi c'est bien parce que tu nous a déjà transmis euh

L2 ce que je vais donner comme argent aux subdivisions

L1 ce que tu vas donner comme argent aux subdivisions

L2 donc tu sais déjà

L1 est en train de partager des informations et s'adresse à l'ensemble des huit participants à la réunion mais son regard est orienté vers L2 lors qu'il produit le premier tour de cet extrait. Il s'adresse même directement à L2 en utilisant la deuxième personne du singulier puis il produit une hésitation (« euh ») qui va favioriser l'intervention complétive de L2. Ce dernier est tout à fait légitime pour compléter et aider son interlocuteur dans la mesure où il peut apporter l'information que L1 souhaite transmettre puisque cela son concerne sa propre activité professionnelle. L2 intervient ici en tant qu'expert Cette complétion est ratifiée par le premier locuteur qui la reprend en l'adaptant d'un point de vue énonciatif. 
Ainsi, les énonciations conjointes mettent au jour les traces de la construction collaborative du discours entre les locuteurs. Elles permettent d'observer les ajustements situationnels et communicationnels caractéristiques des interactions verbales que nous avons mentionnés plus haut. L'analyse des énonciations conjointes montre également que certains locuteurs sont plus à même que d'autres à produire la complétion. Ces locuteurs spécifiques sont légitimes pour particiciper à la construction collaborative de certaines activtiés langagières en raison de leur identité, de leurs connaissances, de leur implication dans la thématique abordée, de leur statut ou de leur rôle dans les interactions. En outre, les locuteurs qui complètent peuvent se servir de la complétion comme une occasion de mettre au jour leur position particulière dans les interactions, une position d'expert, par exemple. La complétion montre alors, par une intervention dans l'élaboration du discours commun, qu'un locuteur est capable d'intervenir sur le sujet en cours.

\subsection{La complétion par anticipation}

L'apparition de la complétion dans une énonciation conjointe peut également ne pas être sollicitée par le premier locuteur ou ne pas être perçue comme une sollicitation de la part du premier locuteur envers le deuxième locuteur. Elle peut être le fruit de l'anticipation d'un locuteur. Dans l'exemple 7, L4 ne produit pas d'indice d'hésitation ou d'indice d'un discours incomplet mais son intervention est tout de même complétée par un interlocuteur.

Exemple 7 (L4 fait part aux autres membres d'un conseil municipal d'un projet de repas réunissant tous les membres du village nés une année se terminant par le chiffre 9. Ce projet fait suite à un autre repas n'ayant pas réuni beaucoup de personnes.)

L4 parce que j'ai relevé la liste il y en a pas mal quand même de chaque catégorie et on s'est trouvé que ça enfin je sais pas moi j'ai pensé que ce serait un moyen de [rire] de mélanger un peu $\backslash+$

L1 les générations

L4 les générations et faire venir peut-être des gens qui bon faire bouger un peu les gens parce que apparemment euh ouh c'est galère hein

L4 semble avoir terminé son unité de construction de tour, il termine d'ailleurs par une intonation descendante et marque une pause. Néanmoins, L1 poursuit la construction syntaxique de L4 pour produire l'énoncé « de mélanger un peu les générations ». La complétion «les générations » est ratifiée par une reprise qui permet à L4 de reprendre son tour et de poursuivre son discours. L1 apporte un ajout d'information grâce à sa complétion mais rien ne laisse penser que L4 ait eu l'intention d'apporter cette information.

Nous retrouvons ce type de complétion dans l'exemple suivant extrait de l'assemblée générale d'une association.

\section{Exemple 8}

L7 non non je ne vais pas parler des cents des cents euros non de non ben moi ce Madame la Présidente hein [rire] ben moi je voudrais vous féliciter déjà vous êtes nombreux parce que euh hein tu disais tout à l'heure que finalement il n'y avait pas mais je crois bien que c'est la première fois à l'assemblée générale de la *A1* où il y a autant de monde hein ou alors euh ça remonte déjà à loin loin je sais pas si c'est

L8 l'effet Beaujolais + [rires] 
L7 j'ai pas dit que c'était le Beaujolais hein + [rires]

Même si le premier tour de parole de L7 est inachevé d'un point de vue syntaxique, il n'est pas certain que cette construction ait été achevée syntaxiquement par ce même locuteur s'il n'avait pas été suivi du tour de parole de L8. Les études sur le français parlé ont largement montré que les locuteurs peuvent laisser volontairement des énoncés inachevés (Blanche-Benveniste, 2000). Les inachèvements ne nuisent pas nécessairement à l'élaboration et à la compréhension du discours. Ils peuvent avoir les effets pragmatiques excomptés par le locuteur bien que toutes les places syntaxiques attendues ne soient pas remplies ou encore ils peuvent laisser ouvertes différentes interprétations possibles, de la suite de l'énoncé et du sens de celui-ci, pour les interlocuteurs. Les inachèvements peuvent également être des stratégies, utilisées par les locuteurs, pour faire référence à un sujet sans le nommer explicitement.

Dans cet exemple, L8 apporte une contribution en complétant syntaxiquement la construction de son interlocuteur L7 avec «l'effet Beaujolais». L'assemblée générale ayant prévu une dégustation de Beaujolais à la fin de sa tenue, L8 fait le lien entre le nombre important de participants et cette dégustation. Néanmoins, la complétion n'est pas ratifiée par L7, elle est rejetée dans l'intervention suivante («j'ai pas dit que c'était le Beaujolais hein »). Cette réaction à la complétion de L8, suivie des rires de l'ensemble des participants, peut signifier: soit que L7 n'interprète pas la présence d'un grand nombre de participants comme étant liée à la dégustation de Beaujolais; soit que L7 n'a pas « dit » qu'il y avait un lien entre les deux, c'est-à-dire qu'il ne l'avait pas verbalisé. Ainsi, même si le discours se construit de manière collaborative avec une énonciation conjointe, cette collaboration n'est pas nécessairement une marque d'accord entre les locuteurs ${ }^{4}$. L'intervention de L8 dans cet exemple montre davantage la connivence entre les locuteurs qui sont tous les membres de l'association réunie en assemblée générale.

L'apparition d'une complétion par anticipation peut trouver une explication dans le statut des locuteurs engagés dans les interactions, comme nous l'avons déjà constaté dans le point précédent. Dans l'exemple 9, L1 est le directeur de l'entreprise (appelée Parc dans l'extrait suivant), il assiste, comme les autres participants, à l'exposé de L9, responsable de la comptabilité. Néanmoins, L1 anticipe la fin d'un tour de paroles de L9.

Exemple 9 : Le responsable de la comptabilité L9 fait référence à une formation à un logiciel de gestion et de comptabilité.

L9 de toute façon les consultes j’avais prévu de faire au Parc $<* C^{*}$ \{numéro du département

L1 une formation $\backslash>$

L9 une formation $\backslash$ je vais la programmer pour septembre $\backslash$

La complétion de L1 permet à ce dernier de se positionner comme supérieur hiérarchique annonçant luimême la tenue d'une formation. L'entreprise s'étant doté d'un nouveau logiciel de comptabilité, une formation est nécessaire pour que les salariés (ici, l'encadrement) puissent consulter ce logiciel (c'estdire-dire faire des «consultes »). L1 interrompt L9 pour anticiper la fin de son unité de tour (il produit la complétion en même temps que le numéro du département produit par L9). La complétion de L1 « une formation » s'inscrit dans la construction syntaxique proposée par L9 et anticipe l'achèvement de l'énoncé de L9. En effet, nous pouvons faire l'hypothèse que L9 était sur le point d'annoncer « une formation », qu'il allait programmer. Cependant, la construction syntaxique de son énoncé facilite l'intervention de L1 qui prend les devants en annonçant, en tant que directeur, la formation. Plus précisément, L9 produit à la suite du verbe « faire » une information géographique « au Parc * $\mathrm{C}^{*}$ » connue de tous les participants. L'introduction d'une précision concernant le lieu permet au supérieur hiérarchique d'être le premier à annoncer la mise en place d' « une formation ». La complétion est alors 
utilisée par L1 comme une ressource interactionnelle permettant de prendre la parole et de souligner sa place hiérarchique. Ensuite, L9 répète la complétion de L1 et poursuit son discours.

Ainsi, l'énonciation conjointe met en lumière des phénomènes interactionnels qui reflètent certains éléments situationnels, par exemple le statut hiérarchique d'un locteur, comme c'est le cas dans l'exemple ci-dessous, ou la relation entre les locuteurs, comme c'est le cas dans l'exemple 8. La complétion par anticipation permet à la fois aux locuteurs de collaborer activement au discours et parfois de se positionner dans le discours en collaborant à la construction de celui-ci. Toutes les énonciations conjointes dont la complétion est produite par anticipation ne révèlent pas des facteurs extralinguistiques particuliers, c'est le cas de l'exemple 1, mais elles sont tout de même des traces de la collaboration ainsi que des ressources pour collaborer au discours.

\section{Conclusion}

L'approche sociolinguistique des interactions verbales permet de saisir, dans un genre de discours particulier, la réalisation des activités langagières grâce à la production de certaines pratiques langagières. Dans le cadre de cette étude, nous avons souhaité analyser des pratiques langagières collaboratives, les énonciations conjointes, actualisées à la fois pour réaliser des activités langagières et pour produire certains effets pragmatiques. En d'autres termes, les énonciations conjointes peuvent être analysées comme des traces de la construction collaborative du discours dans la mesure où elles montrent les modes de production d'un discours commun produit à plusieurs. Ce phénomène collaboratif est également utilisé comme une ressource interactionnelle par certains locuteurs pour mettre au jour des éléments de la situation de communication, tels que leur identité, leur statut, leurs connaissances, leur relation avec les autres participants ou encore leur engagement conjoint dans l'élaboration du discours. Nos analyses des énonciations conjointes prennent leur source dans les liens entre le discours des locuteurs et les éléments extralinguistiques nous renseignant sur la situation de communication.

\section{Références bibliographiques}

André, V. (2011). «Ton corpus j’y comprends rien!». De la difficulté de partager des données orales. Colloque AFLS. Regards nouveaux sur les liens entre théories, méthodes et données en linguistique française, Nancy, les 8-10 septembre.

André, V. (2006). Construction collaborative du discours au sein de réunions de travail en entreprise. De l'analyse micro-linguistique à l'analyse socio-interactionnelle. Thèse de doctorat. Sous la direction de P. Riley. Université Nancy 2.

André, V., Canut, E. (2010). Mise à disposition de corpus oraux interactifs : le projet TCOF (Traitement de Corpus Oraux en Français), Pratiques, 147-148, 35-51.

André-Larochebouvy, D. (1984). La conversation quotidienne. Introduction à l'analyse sémio-linguistique de la conversation, Paris : Didier.

Aston, G. (1988). Negociating Service, Bologna : CLUEB.

Blanche-Benveniste, C. (2000). Approche de la langue parlée en français, Paris : Ophrys. 
Blanche-Benveniste, C., Jeanjean, C. (1987). Le français parlé, Paris : Didier Eruditions.

Boutet, J. (1998). Aspects linguistiques, ergonomiques et didactiques de la parole au travail. In Lazar, A. (éd.), Langage(s) et travail : Enjeux de formation. Actes du colloque INRP/CNAM/CNRS-LT, Octobre 1998, Paris : INRP, 75-78.

Charaudeau, P. (1995). Rôles sociaux et rôles langagiers. In Véronique, D., Vion, R., Modèles de l'interaction verbale, Aix-en-Provence : Publications de l'Université de Provence, 79-96.

Clark, H. H. (1996). Using Language, Cambridge : Cambridge University Press.

Coastes, J. (1994). No gap, lots of overlap : turn-taking patterns in the talk of women friends. In Graddol, D., Maybin, J., Stierer, B. (Eds.), Researching language and Literacy in Social Context. Multilingual Matters, Clevedon: Open University, 177-192.

Culioli, A. (1990-1999). Pour une linguistique de l'énonciation. 3 Tomes, Paris : Ophrys.

Falk, J. (1980). The conversational duet. Proceedings of the 6th Annual Meeting of the Berkeley Linguistics Society, University of California: Berkeley, 507-514.

Gumperz, J. (1982). Discourse Strategies, Cambridge: Cambridge University Press.

Heritage, J. (1995). Conversation Analysis. Methodological aspects. In Quasthoff, U. M. (Eds), Aspects of Oral Communication, New York : Walter de Gruyter, 391-418.

Hutchins, E. (1995). Cognition in the wild, Cambridge : MIT Presse.

Hymes, D. (1972). Models of the interaction of language and social life. In Gumperz, J., Hymes, D. (Eds), Directions in Sociolinguistics. The Ethnography of Communication, Oxford : Basil Blackwell, 35-71.

Jeanneret, T. (1995). Conversations pluri-locuteurs et co-énonciation. In Véronique, D., Vion, R. (Eds), Modèles de l'interaction verbale. Aix-en-Provence : Presses Universitaires de Provence, 379-390.

Jeanneret, T. (1999). La coénonciation en français, Bern : Peter Lang.

Jefferson, G. (1973). A Case of Precision Timing in Ordinary Conversation : Overlapped Tag-Positioned Address Terms in Closing Sequences. Semiotica, 9, 47-96.

Kerbrat-Orecchioni, C. (2005). Le discours en interaction. Paris : Armand Colin.

Lonchamp, J. (2003). Le travail coopératif et ses technologies. Paris : Lavoisier.

Loufrani, C. (1981). Le locuteur collectif ou locuteur tout court. Recherches sur le français parlé, 3, 215-243.

Loufrani, C. (1985). Le locuteur collectif : typologie de configurations discursives. Recherches sur le français parlé, 6, 169-193.

Maury-Rouan, C. (1998). Le parallélisme co-énonciatif. Construire à plusieurs l'allocutaire absent : l'énonciateur 'en creux' dans le dialogue. Revue de Sémantique et de Pragmatique, 3, 145-158.

Mondada, L. (1999a). L'organisation séquentielle des ressources linguistiques dans l'élaboration collective des descriptions. Langage et société, 89, 9-36.

Mondada, L. (1999b). Formes de séquentialité dans les courriels et les forums de discussion. ALSIC, Vol. 2, Numéro 1, 3-25. Disponible sur: http://alsic.u-strasbg.fr.

Sacks, H. (1992). Lectures on Conversation. Edited by Gail Jefferson. (1964-1972). Oxford: Basil Blackwell, 2 volumes.

Sacks, H., Schegloff, E., Jefferson, G. (1974). A simplest systematics for the organization of turn-taking in conversation. Language, 50, 696-735.

\section{Conventions de transcription}


Les conventions de transcription sont celles du projet TCOF (http://www.cnrtl.fr/corpus/tcof/TCOFConventions.pdf) et sont enrichies pour les besoins de l'analyse.

$<\ldots>\quad$ chevauchement de paroles

$:: \quad$ allongement de syllabe

+ pause

*P* $\quad$ anonymisation d'un patronyme

*A* anonymisation du nom d'une association

${ }^{*} \mathrm{C}^{*} \quad$ anonymisation d'un chiffre ou d'un nombre

$\{\ldots\} \quad$ commentaire

...- bribe de mot

/ intonation montante

1 intonation descendante

1 Le terme «éclectique» étant souvent connoté péjorativement en français, l'expression «synchrétisme méthodologique » est également utilisée (par exemple Aston, 1988 ; Heritage, 1995).

${ }^{2}$ Disponible à l'adresse : $\underline{\text { http://trans.sourceforge.net }}$

${ }^{3}$ Voir les conventions de transcription précisées à la fin de cet article.

${ }^{4}$ Notre analyse de la construction collaborative au sein de réunions de travail montre que les énonciations conjointes peuvent être actualisées stratégiquement pour mettre en évidence un accord entre certains participants (André, 2006). Dans ces cas, les énonciations conjointes sont non seulement des ressources et des traces de collaboration entre les locuteurs mais elles sont également les marques des alliances entre les participants. 